\title{
A new family with a homozygous nonsense variant in NTHL1 further delineated the clinical phenotype of NTHL1-associated polyposis
}

\author{
Mays Altaraihi', Anne-Marie Gerdes ${ }^{1}$ and Karin Wadt [1]
}

\begin{abstract}
A new family with NTHL1-associated polyposis (NAP) is described, involving a 58-year-old male affected with $>100$ colorectal polyps and a 55-year-old female sibling with nine colorectal polyps. The female was also diagnosed with a thyroid adenoma at age 40 . Significantly, no malignant neoplasms have been detected in this family, which is important to further delineate the clinical phenotype related to NAP. A review of previously reported obligate heterozygous carriers of NTHL 1 variants showed two patients affected with neoplasms at $<55$ years of age, generating a study to outline the phenotypic spectrum in patients with heterozygous pathogenic NTHL1 variants relevant.
\end{abstract}

Colorectal adenomatous polyposis syndromes can be divided into familial adenomatous polyposis (FAP), $M U T Y H$-associated polyposis (MAP), polymerase proofreading-associated polyposis (PPAP) and NTHL1associated polyposis (NAP).

FAP is responsible for approximately $1 \%$ of all $\mathrm{CRCs}^{1}$ and is due to pathogenic variants in the adenomatous polyposis coli $(A P C)$ gene. The APC gene is the most frequently mutated gene associated with colorectal adenomatous polyposis. The MUTYH gene, which is involved in the base excision repair (BER) pathway, is the second most commonly mutated gene associated with adenomatous polyposis. In contrast to FAP and PPAP, MAP shows recessive inheritance.

Recently, Weren et al. uncovered another gene in the BER pathway, NTHL1, associated with adenomatous polyposis and recessive inheritance ${ }^{2}$. Apart from adenomatous polyposis, the occurrence of ovarian cancer, duodenal cancer, basal cell carcinoma, breast cancer, endometrial malignancy, meningioma, and bladder cancer have been reported in individuals with loss-of-function variants in $N T H L 1^{3}$.

\footnotetext{
Correspondence: Karin Wadt (karin.wadt@regionh.dk)

${ }^{1}$ Department of Clinical Genetics, Copenhagen University Hospital

Rigshospitalet, Copenhagen, Denmark
}

We report a new family with NAP:

\section{Patient 1}

A 47-year-old man (patient 1) developed hematochezia triggered by a gastrointestinal infection (Fig. 1). A colonoscopy was performed, identifying 23 polyps; three in the caecum, the rest in the left colon. Polypectomy was performed, and a pathology review of eight polyps showed six tubular adenomas with either low or moderate dysplasia and two tubulovillous adenomas, one of which had severe dysplasia. The largest adenoma measured $20 \mathrm{~mm} \times$ $8 \mathrm{~mm} \times 15 \mathrm{~mm}$.

The patient had no family history of colonic adenomas or cancer, and genetic testing was not performed. However, the patient was enrolled for routine screening, and during a 10-year span (2007-2017), annual colonoscopic polypectomies identified and removed approximately 40 polyps. Approximately eighty percent of the polyps were adenomas.

In June 2018, at age 58, a colonoscopy was performed, identifying and removing 55 new polyps that had developed during the preceding 6 months. Four of the five pathologically described polyps were adenomas; the last polyp was identified as a sessile serrated polyp.

\section{(c) The Author(s) 2019}

(c) (i) Open Access This article is licensed under a Creative Commons Attribution 4.0 International License, which permits use, sharing, adaptation, distribution and reproduction cc) in any medium or format, as long as you give appropriate credit to the original author(s) and the source, provide a link to the Creative Commons license, and indicate if changes were made. The images or other third party material in this article are included in the article's Creative Commons license, unless indicated otherwise in a credit line to the material. If material is not included in the article's Creative Commons license and your intended use is not permitted by statutory regulation or exceeds the permitted use, you will need to obtain permission directly from the copyright holder. To view a copy of this license, visit http://creativecommons.org/licenses/by/4.0/. 


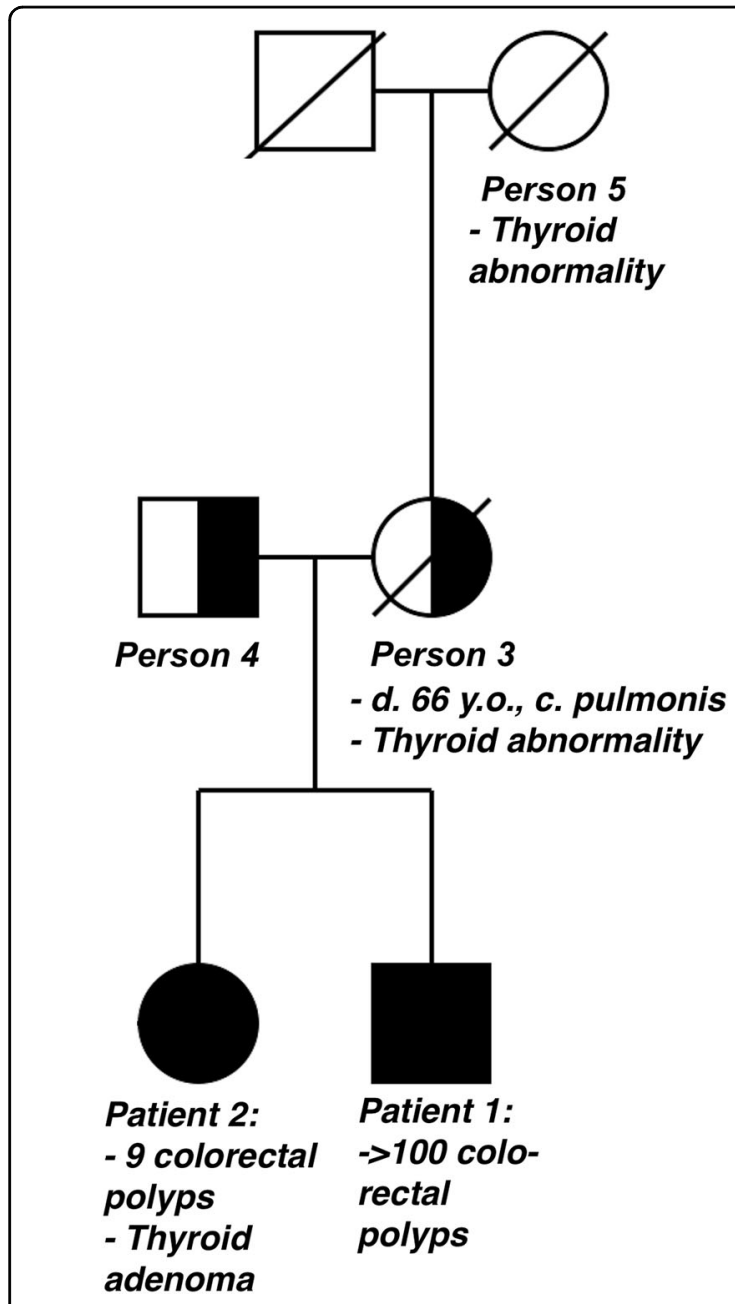

Fig. 1 The pedigree shows the first identified Danish family carrying a homozygous nonsense variant in NTHL1

The most recent colonoscopy, performed in December 2018, showed 20 polyps, in which all pathologically described polyps were tubular adenomas with low dysplasia. The patient has developed 133 polyps so far and has declined undergoing a recommended colectomy. $\mathrm{He}$ was referred to genetic counseling, and an analysis of a colorectal cancer gene panel consisting of 17 genes was performed (APC, BMPR1A, CDH1, EPCAM, GREM1, MLH1, MSH2, MSH6, MUTYH, NTHL1, PMS2, POLD1, POLE, PTEN, SMAD4, STK11, TP53). A previously reported homozygous nonsense variant (NM_002528): c. $268 \mathrm{C}>\mathrm{T}$, p. Gln90Ter in the NTHL1 gene was identified. No alterations were found in the other examined genes.

As other cancer types have been described in patients with homozygous nonsense variants in NTHL1, a gastroscopy and a dermatological examination were performed, finding no suspicious results. A urine cytology test showed no malignant cells.

\section{Patient 2}

The 55-year-old sister of patient 1 presented with a thyroid adenoma at age 40 , which was later characterized as a cold nodule.

Due to multiple colorectal polyps in her brother, the patient underwent a colonoscopy, and a tubular adenoma with moderate dysplasia was removed from the caecum at age 45. She was referred to genetic counseling, and genetic testing identified the same homozygous nonsense variant, c.268C $>\mathrm{T}$, in NTHL1.

A gastroscopy and a dermatological examination revealed no suspicious findings.

A recent colonoscopy showed eight colorectal polyps, which were removed. Four polyps were pathologically reviewed, classifying three as tubular adenomas with low dysplasia and one sessile serrated polyp. Mammography and gynecological examination were conducted, revealing no signs of breast or endometrial cancer, respectively.

\section{Other family members}

Person 3, the mother of patients 1 and 2, is an obligate heterozygous carrier of the nonsense variant, c. $268 \mathrm{C}>\mathrm{T}$, in NTHL1. At age 58, she was diagnosed with pulmonary squamous cell carcinoma, having a smoking history of 67.5 pack-years. She had also undergone thyroidectomy due to goiter, approximately at age 50 . The patient died of lung cancer at 66 years old.

Person 4, the father of patients 1 and 2, is also an obligate heterozygous carrier of the NTHL1 variant and has no neoplasms or thyroid-associated diseases.

The offspring of patients 1 and 2 are obligate heterozygous carriers and are all healthy.

This study reports the first family with a homozygous nonsense variant in the NTHL1 gene in Denmark. Globally, 18 families (30 individuals) with biallelic NTHL1 variants have been reported ${ }^{4}$. The majority $(N=23)$ of the reported patients are homozygous for the same nonsense variant: c. $268 \mathrm{C}>\mathrm{T}$. Five of the reported patients are compound heterozygous individuals. Four of them have the c. $268 \mathrm{C}>\mathrm{T}$, NTHL1 variant (Table 1 in ref. ${ }^{5}$ ). c. $268 \mathrm{C}>\mathrm{T}$ is the most frequent lossof-function variant in NTHL1, with an allele count of 400 (allele frequency of $0.14 \%$ ) according to gnomAD browser

( $N=141,111$ exomes), all in a heterozygous state, with the highest frequency in the European Finnish population (0.35\%) (http://gnomad.broadinstitute.org/variant/ 16-2096239-G-A).

This family further expands the phenotypic spectrum linked to NTHL1-associated polyposis and raises the total number of families to 19 (32 individuals).

Previously, one individual with biallelic variants in NTHL1 has been reported with thyroid cancer, diagnosed at age $60^{5}$. Patient 2 in this study, a homozygous carrier of 
the pathogenic NTHL1 variant, developed thyroid adenoma at age 40. Her mother (person 3 ) had undergone a thyroidectomy due to a thyroid abnormality. No specification of the thyroid abnormality can be made as no medical records or tissue from the thyroidectomy of person 3 can be found.

Papillary thyroid cancer, thyroid nodules, and multinodular goiter have been reported in patients with FAP and $\mathrm{MAP}^{6,7}$, suggesting a link between thyroid abnormalities and NAP, although disorders of the thyroid gland can be associated with several other genetic diseases, which has not been analyzed in this family.

Moreover, studies have shown that heterozygous carriers of pathogenic MUTYH variants have twice the population risk of developing colorectal adenomas and endometrial cancer and three times the population risk of developing liver and gastric cancer ${ }^{8}$. One study suggested no increased risk of developing colorectal adenomas in individuals heterozygous for the pathogenic NTHL1 variant $^{9}$. None of the heterozygous carriers in the current family had a colonoscopy performed.

However, reported manifestations found in obligate NTHL1 heterozygous carriers in the literature may suggest a heightened risk of developing other neoplasms. Significantly, one female was diagnosed with an endometrial tumor at age 41 , and one male was diagnosed with gastric cancer at age 53 . One heterozygous female carrier of the c. $268 \mathrm{C}>\mathrm{T}$ variant was diagnosed with two undescribed tumors. The number of NTHL1 families is very limited, and only a few NTHL1 heterozygous patients are reported with symptoms, so no definite conclusion can be drawn.

Patient 1 developed 133 colorectal polyps by the age of 58,55 of which developed during a 6 -month period. This observation is important when considering the natural history of colorectal polyposis associated with NTHL1 mutations and corroborates the current recommendation of colectomy when a considerable number of adenomas are identified. This case report is the second to describe a patient with a pathogenic homozygous NTHL1 variant affected with $>100$ colorectal polyps, both being males ${ }^{10}$. Previously, only four reported biallelic NTHL1 variant carriers have been described without malignant neoplasms, raising the number to six out of 32 patients without any malignant neoplasms when including the siblings. This observation is certainly age-dependent, and both reported patients have also been under clinical surveillance with short intervals, which may contribute to finding no malignant neoplasms.

In conclusion, none of the reported siblings, aged 55 and 58 years, developed malignant neoplasms; however, the median age at diagnosis for colorectal cancer in NTHL1 homozygous or compound heterozygous carriers is 59 years for men and 64 years for women ${ }^{5}$.

Moreover, we have observed thyroid abnormalities in both heterozygote and homozygote carriers in this family. Studies of larger cohorts of individuals with NTHL1 variants in both heterozygous and homozygous states can further delineate the phenotypic spectrum.

\section{HGV Database}

The relevant data from this Data Report are hosted at the Human Genome Variation Database at https://doi.org/10.6084/m9.figshare.hgv.2606

Conflict of interest

KW has participated in an advisory board meeting for MSD.

\section{Publisher's note}

Springer Nature remains neutral with regard to jurisdictional claims in published maps and institutional affiliations.

Received: 15 April 2019 Revised: 30 July 2019 Accepted: 1 August 2019. Published online: 10 October 2019

\section{References}

1. Fearnhead, N. S., Britton, M. P. \& Bodmer, W. F. The ABC of APC. Hum. Mol. Genet. 10, 721-733 (2001).

2. Weren, R. D. et al. A germline homozygous mutation in the base-excision repair gene NTHL1 causes adenomatous polyposis and colorectal cancer. Nat. Genet. 47, 668-671 (2015).

3. Rivera, B., Castellsague, E., Bah, I., van Kempen, L. C. \& Foulkes, W. D. Biallelic NTHL1 Mutations in a Woman with Multiple Primary Tumors. N. Engl. J. Med. 373, 1985-1986 (2015).

4. Groves, A., Gleeson, M. \& Spigelman, A. D. NTHL1-associate polyposis: first Australian case report. Fam. Cancer 18, 179-182 (2019).

5. Grolleman, J. E. et al. Mutational signature analysis reveals NTHL1 deficiency to cause a multi-tumor phenotype. Cancer Cell. 35, 256-266 e5 (2019).

6. Nielsen, M., Lynch, H., Infante, E., Brand, R. MUTYH-Associated Polyposis. (eds Adam MP, Ardinger HH, Pagon RA, Wallace SE, Bean $\sqcup H$, Stephens $K$, et al.) (GeneReviews((R)), Seattle, WA, 1993).

7. Iwama, T. et al. Somatic mutation of the APC gene in thyroid carcinoma associated with familial adenomatous polyposis. Jpn J. Cancer Res. 90, 372-376 (1999).

8. Win, A. K. et al. Cancer risks for monoallelic MUTYH mutation carriers with a family history of colorectal cancer. Int J. Cancer 129, 2256-2262 (2011).

9. Belhadj, S. et al. Delineating the Phenotypic Spectrum of the NTHL1Associated Polyposis. Clin. Gastroenterol. Hepatol. 15, 461-462 (2017).

10. Fostira, F. et al. Extending the clinical phenotype associated with biallelic NTHL1 germline mutations. Clin. Genet. 94, 588-589 (2018). 